\title{
Water-Rock Differentiation of Icy Bodies by Darcy law, Stokes law, and Two-Phase Flow
}

\author{
Wladimir Neumann, Doris Breuer and Tilman Spohn
}

Deutsches Zentrum für Luft- und Raumfahrt (DLR), Institut für Planetenforschung, Planetenphysik, Rutherfordstr. 2, 12489 Berlin, Germany, email: wladimir.neumann@dlr.de

\begin{abstract}
The early Solar system produced a variety of bodies with different properties. Among the small bodies, objects that contain notable amounts of water ice are of particular interest. Water-rock separation on such worlds is probable and has been confirmed in some cases. We couple accretion and water-rock separation in a numerical model. The model is applicable to Ceres, icy satellites, and Kuiper belt objects, and is suited to assess the thermal metamorphism of the interior and the present-day internal structures. The relative amount of ice determines the differentiation regime according to porous flow or Stokes flow. Porous flow considers differentiation in a rock matrix with a small degree of ice melting and is typically modelled either with the Darcy law or two-phase flow. We find that for small icy bodies two-phase flow differs from the Darcy law. Velocities derived from two-phase flow are at least one order of magnitude smaller than Darcy velocities. The latter do not account for the matrix resistance against the deformation and overestimate the separation velocity. In the Stokes regime that should be used for large ice fractions, differentiation is at least four orders of magnitude faster than porous flow with the parameters used here.
\end{abstract}

Keywords. Dwarf planets, Asteroids, Planetesimals, Differentiation, Accretion, Ceres

\section{Introduction}

Objects with an ice-silicate composition (comets, asteroids, and dwarf planets) can be found in the outer asteroid belt (e.g., the dwarf planet Ceres) and populate the outer range of the Solar system. We present a numerical model that calculates the evolution of small icy bodies and combines accretion and differentiation into a rocky core and a water mantle (and further possible metal-silicate separation of the rocky core). To simulate differentiation, we use a multiphase model for which phase changes and separation by matrix compaction are taken into account in spherical symmetry, considering simultaneously ice and rock in solid and liquid states. The model provides a numerical solution of a moving boundary problem for the temperature $T(r, t)$ as a function of radius $r$ and time $t$ on a moving ("accreting") domain of a planetesimal's interior. In particular, we compare the theoretical predictions based on Darcy and Stokes law with the differentiation velocities calculated with the numerical model. The basic structure of differential equations is given below and is built upon the thermal evolution and accretion code from Srámek et al. (2012) (the system of equations we use here, however, is applied to an icy body). The model is designed to address the evolution of Ceres, but it is applicable to any small icy object.

\section{Model}

The model calculates the thermal evolution and differentiation in 1D for an object that consists of ice and dust and has the size and the density of Ceres. The separation of water and rock is modelled within the formalism of the two-phase flow theory. Thereby, the modelled processes are coupled with the continuous growth of the asteroid due to the accretion of dust. 
Frame of reference and accretion: All differential equations involved are transformed from the formulations on $(r, t)$ with the radial coordinate $r$ and time $t$ to those on a moving frame $(\eta, t): \eta:=r \cdot R(t)^{-1}$, where $R(t)$ is the radius evolution. Here, we only present models of instantaneous formation with $R(t)=R_{0}=R_{f}=480 \mathrm{~km}$ (with the initial radius $R_{0}$ and the final radius $R_{f}$ ).

Energy balance: Energy balance equation is solved with parameters that depend on temperature, porosity and composition (with bulk density $\rho$, space coordinate $\eta$, bulk heat capacity $c_{p}$, temperature $T$, current radius of the planetesimal $R$, bulk thermal conductivity $k$, heat sources $Q$, latent heat $L$, melting rate $\Gamma$, water $w$ and dust $d$ ):

$$
\rho(\eta) c_{p}(\eta, T)\left(\frac{\partial T}{\partial t}-\eta \frac{\dot{R}}{R} \frac{\partial T}{\partial \eta}\right)=\frac{1}{\eta^{2}} \frac{\partial}{\partial \eta}\left(\frac{k(\eta)}{R^{2}} \eta^{2} \frac{\partial T}{\partial \eta}\right)+Q(\eta, t)-L_{w} \Gamma_{w}-L_{d} \Gamma_{d} .
$$

Water-rock and metal-silicate differentiation: Water percolation / particle settling is modelled by solving advection equations for the matrix and the liquid phases within the twophase flow formalism (with solid fraction $\phi_{s}^{i}$ or liquid fraction $\phi_{l}^{i}$ of component $i$, index $i$ equal to $d$ or $w$, average separation velocity $v(\eta)$, and density $\rho_{i}$ of component $i$ ):

$$
\frac{\partial \phi_{s}^{i}}{\partial t}-\eta \frac{\dot{R}}{R} \frac{\partial \phi_{s}^{i}}{\partial \eta}+\frac{1}{R \eta^{2}} \frac{\partial}{\partial \eta}\left(\eta^{2} \phi_{s}^{i} v\right)=-\frac{\Gamma_{i}}{\rho_{i}}, \quad \frac{\partial \phi_{l}^{i}}{\partial t}-\eta \frac{\dot{R}}{R} \frac{\partial \phi_{l}^{i}}{\partial \eta}+\frac{1}{R \eta^{2}} \frac{\partial}{\partial \eta}\left(\eta^{2} \phi_{l}^{i} v\right)=-\frac{\Gamma_{i}}{\rho_{i}} .
$$

After the separation of water, the solidus of metal can be reached. Then, the same equations can be used for the components $F e$ (metal) and $S i$ (silicates) with the respective densities and melting rates.

Differentiation velocity: The water percolation velocity is computed according to the twophase flow theory (with viscosities of the liquid $\mu_{l}$ and of the solid $\mu_{s}$, permeability $K_{\phi}$, fraction of the liquid $\phi_{l}=\phi_{l}^{w}+\phi_{l}^{d}$, fraction of the solid $\phi_{s}=\phi_{s}^{w}+\phi_{s}^{d}$, critical liquid fraction $\phi_{c}$, and gravity $g$ ):

$$
\frac{\mu_{l}}{K_{\phi}} v=\frac{1}{R^{2}} \frac{4}{3} \mu_{s} \frac{\partial}{\partial \eta}\left(\frac{\phi_{s}}{\eta^{2}} \frac{\partial}{\partial \eta} \eta^{2} v\right)+\frac{1}{R^{2}} \frac{\partial}{\partial \eta}\left(\frac{\mu_{s} \phi_{s}}{\phi_{l}-\phi_{c}} \frac{1}{\eta^{2}} \frac{\partial}{\partial \eta} \eta^{2} v\right)+\frac{4 \mu_{s}}{R^{2}} \frac{\partial \phi_{l}}{\partial \eta}-\left(\phi_{s}^{d}+\phi_{l}^{d}\right)\left(\rho_{d}-\rho_{w}\right) g .
$$

For the metal-silicate separation the velocity is computed analogously using suitable parameters. The permeability is computed from the reference permeability $K_{0}$ at the reference liquid fraction $\phi_{0}$ (equal to initial volume fraction of ice), with $K_{0}=b^{2} / \tau$, solid grain size $b$, coefficient $\tau=72 \pi$, and exponent $n=2$ :

$$
K_{\phi}=K_{0}\left(\phi_{l}^{w}-\phi_{c}\right)^{n} /\left(\phi_{0}-\phi_{c}\right)^{n} \text {. }
$$

Material properties: A mixture of ice and dust is considered. The initial mass fraction of dust is $0.865-0.737$ (ice mass fractions of $0.135-0.263$ ), corresponding to the rock density variation of $2540-3450 \mathrm{~kg} \mathrm{~m}^{-3}$ (from a low-density serpentine-like to a high-density Vesta-like material). The associated ice volume fraction is $\phi_{0}=0.284-0.552$. The ice density $\rho_{w}=1000 \mathrm{~kg} \mathrm{~m}^{-3}$ is fixed, the volume-weighted average density of the asteroid is $\rho=2100 \mathrm{~kg} \mathrm{~m}^{-3}$ for the above variation. The thermal conductivity $k$ is volume-weighted arithmetic mean of the heat conductivities of ice $\left(k_{d}=0.4685+488.12 T^{-1}\right)$ and rock $\left(k_{w}=2.2 \mathrm{~W} \mathrm{~m}^{-1} \mathrm{~K}^{-1}\right)$. The specific heat capacity is a mass-weighted arithmetic mean of temperature dependant heat capacities of ice $\left(c_{p, w}=\min (4200,185+7.037 T) \mathrm{J} \mathrm{K}^{-1} \mathrm{~kg}^{-1}\right)$ and rock $\left(c_{p, d}=800+0.25 T-1.5 \times 10^{7} T^{-2} \mathrm{~J} \mathrm{~K}^{-1} \mathrm{~kg}^{-1}\right)$.

Heat sources: Both short- $\left({ }^{26} \mathrm{Al},{ }^{60} \mathrm{Fe},{ }^{53} \mathrm{Mn}\right)$ and long-lived $\left({ }^{40} \mathrm{~K},{ }^{232} \mathrm{Th},{ }^{235} \mathrm{U},{ }^{238} \mathrm{U}\right)$ radiogenic nuclides are included in the rock fraction, while the ice fraction contains no heat sources.

Initial conditions: The initial and surface temperature is $180 \mathrm{~K}$. The surface temperature remains constant, the central heat flux is zero. 
solid rock matrix solid rock matrix

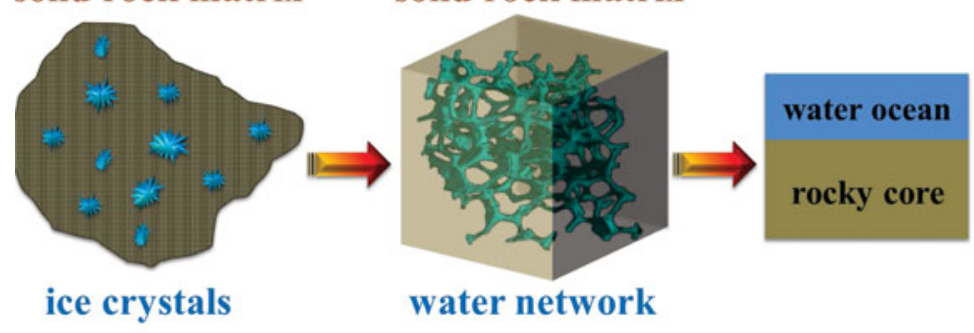

solid ice matrix

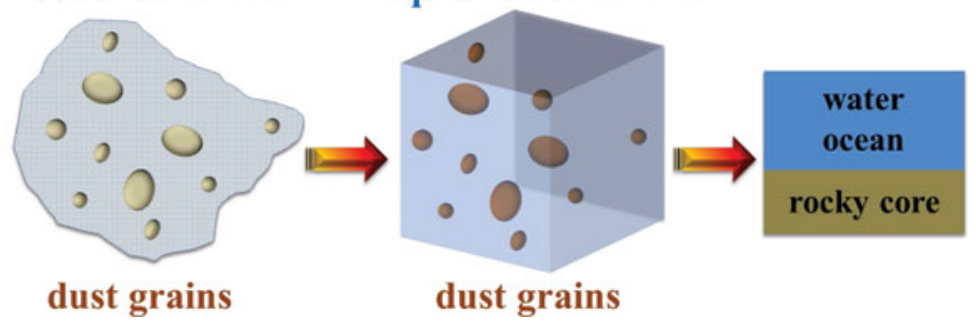

Figure 1. Overview of the differentiation regimes. Top panel: The composition is dominated by the dust. Melting of ice creates a water network. Percolation of water results in the formation of a water ocean and a rocky core. Bottom panel: The composition is dominated by ice. Upon melting of the ice matrix a water ocean forms and a rocky core grows by sinking dust particles.

\section{Results}

In the following, we first describe two differentiation regimes and compare their differentiation velocities. To this end, we consider two extreme ice mass fractions of 0.135 and 0.2629 .

Ice-rock separation regimes: Assuming accretion of ice and dust, the rheology of the matrix is dominated by one of the two components, depending on their relative proportions. Two differentiation regimes arise (see Fig. 2): (a) Upon melting of ice in a rocky matrix water ascends via Darcy flow (Fig. 2, top); (b) Upon melting of an icy matrix the dust grains settle via Stokes flow (Fig. 2, bottom). For (a) water will percolate if the matrix deforms sufficiently to squeeze the water out of the matrix. Because temperatures of up to $700 \mathrm{~K}$ are needed for this, water will first remain immobile until the matrix deforms and then percolate, or until vapour forms and gets mobilised by the matrix deformation. On its way to the surface water and ice will condense or crystallise in cooler layers.

Separation velocity: Estimates of the particle settling velocity and water percolation velocity can be obtained from Stokes and Darcy law, respectively. Free parameters are: Grain size $b$, gravity $g<0.25 \mathrm{~m} \mathrm{~s}^{-2}$ (Ceres' surface gravity), dust density $\left(2500<\rho_{d}<\right.$ $3600 \mathrm{~kg} \mathrm{~m}^{-3}$, see McCord \& Sotin (2005)), permeability parameters $n$ and $\tau$, and liquid volume fraction $\phi_{l}^{w}$. Fixed parameters are water density $\rho_{w}=1000 \mathrm{~kg} \mathrm{~m}^{-3}$ and water viscosity $\eta_{l}=0.002 \mathrm{~Pa}$ s. As an upper bound, a gravity $g=0.25 \mathrm{~m} \mathrm{~s}^{-2}$ is used. Darcy and Stokes velocities $v_{D}$ and $v_{S}$ follow from vspace- $0.2 \mathrm{~cm}$

$$
v_{D}=K_{\phi}\left(\rho_{d}-\rho_{w}\right) g / \phi_{l}^{w} \mu_{l}, \quad v_{S}=2\left(\rho_{d}-\rho_{w}\right) g b^{2} / 9 \mu_{l} .
$$

In Fig. 3, Darcy and Stokes velocities are given as function of $\phi_{l}^{w}$ and $b$. Solid lines show velocity in $\mathrm{m} \mathrm{s}^{-1}$ and dashed lines in $\mathrm{m}_{\text {year }}{ }^{-1}$, blue lines correspond to the dust density of $\rho_{d}=2500 \mathrm{~kg} \mathrm{~m}^{-3}$ (serpentine) and red ones to $\rho_{d}=3600 \mathrm{~kg} \mathrm{~m}^{-3}$ (Vesta-like). The Darcy velocity varies between $10^{-12}$ and $10^{-10} \mathrm{~m} \mathrm{~s}^{-1}$ with $\phi_{l}^{w}=0.01-0.5$ for a fixed grain size of $b=10^{-6} \mathrm{~m}$ (and between $10^{-6}$ and $10^{-4} \mathrm{~m} \mathrm{~s}^{-1}$ for $\phi_{l}^{w}=0.01-0.5$ and 

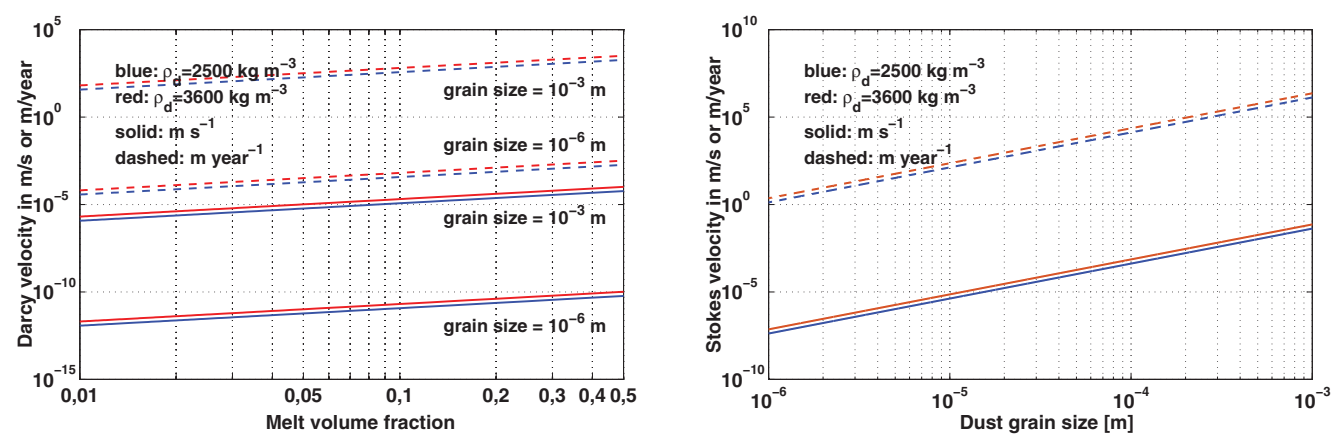

Figure 2. Darcy velocity (left panel) and Stokes velocity (right panel) calculated for $\rho_{d}=2500$ $\mathrm{kg} \mathrm{m}{ }^{-3}$ (blue lines) and $\rho_{d}=3600 \mathrm{~kg} \mathrm{~m}^{-3}$ (red lines) and varying values of $b$ and $\phi_{l}^{w}$.

$b=10^{-3} \mathrm{~m}$ ). The Stokes velocity varies between $10^{-8}$ and $10^{-2} \mathrm{~m} \mathrm{~s}^{-1}$ for grain sizes between $10^{-6}$ and $10^{-3} \mathrm{~m}$. In general, $v_{S}$ is two orders of magnitude higher than $v_{D}$ for an equal grain size. Thus, if the water fraction is low (for instance $\phi_{0}=0.284$ ) liquid water percolates comparatively slow in the Darcy regime. When liquid water is produced rapidly in large amounts before it can percolate upwards (probable for $\phi_{0}=0.552$ ), a muddy water-dust ocean will form. In this case grains settle very fast at the bottom of the ocean on the time scale shown in Fig. 3, right panel. For a serpentine-like matrix, Ceres' differentiation velocity is can be estimated by the ice volume fraction $\left(\phi_{l}^{w}=\phi_{0}=0.284\right)$ and is $\approx 3 \times 10^{-5} \mathrm{~m} \mathrm{~s}^{-1}\left(\approx 1 \mathrm{~km} \mathrm{a}^{-1}\right)$ for $b=10^{-3}$ (Fig. 3, left panel) - differentiation is completed within $\approx 500$ years. For a high density dust fraction, complete melting of ice leads to $\phi_{l}^{w}=\phi_{0}=0.552$. At such liquid fraction Stokes regime occurs. For $b=10^{-3}$, the Stokes velocity is $0.072 \mathrm{~m} \mathrm{~s}^{-1}$ or $\approx 2 \times 10^{6} \mathrm{~m} \mathrm{a}^{-1}$. This extreme velocity implies a complete differentiation of Ceres within less than one year. During the differentiation, the fractions of dust and water and the grain size vary with radius and with time. For this reason, both regimes with the velocity ranges showed in Fig. 3 can occur locally.

Two-phase flow: In addition, we calculate water-rock differentiation of Ceres selfconsistently as described in section 2. As an example, we consider instantaneous formation at $t_{0}=1 \mathrm{Ma}$ relative to the formation of the calcium-aluminium-rich inclusions (CAIs). The viscosity of a serpentine-like matrix is temperature-dependent but not well know and represents an uncertainty in our model. We use $\mu_{s}=10^{18}$ as a lower bound. In addition, the gravity is depth-dependent and the liquid fraction is computed selfconsistently during the differentiation. It can be shown that the separation velocity is not equal to the Darcy velocity, since the resistance of the matrix against the deformation is a counter-acting factor. The velocities calculated for both matrix densities are shown in Fig. 3. Values obtained for the grain size $b=10^{-3}$ are at most $\approx 2.5 \times 10^{-6}$ and are, thus, smaller than the Darcy velocity of $\approx 4 \times 10^{-6}$ for $\phi_{l}^{w}=0.02$. The timing of the core and ocean formation is similar for both dust composition and varies around $10-20$ thousand years. The Darcy law overestimates the differentiation velocity for $\mu_{s}=10^{18}$ since it does not account for the resistance of the matrix against the deformation. Because we use a rather small value for the viscosity of the serpentine matrix, even a slower core and ocean formation is probable. Nevertheless, the phases separate very fast compared to the time scale of the decay of radiogenic nuclides, leading to a complete separation of water from the dust. Only a very thin undifferentiated layer remains at the surface. The water layer is $\approx 50 \mathrm{~km}$ thick for a serpentine-like dust composition and $\approx 112 \mathrm{~km}$ for a Vesta-like dust composition. 

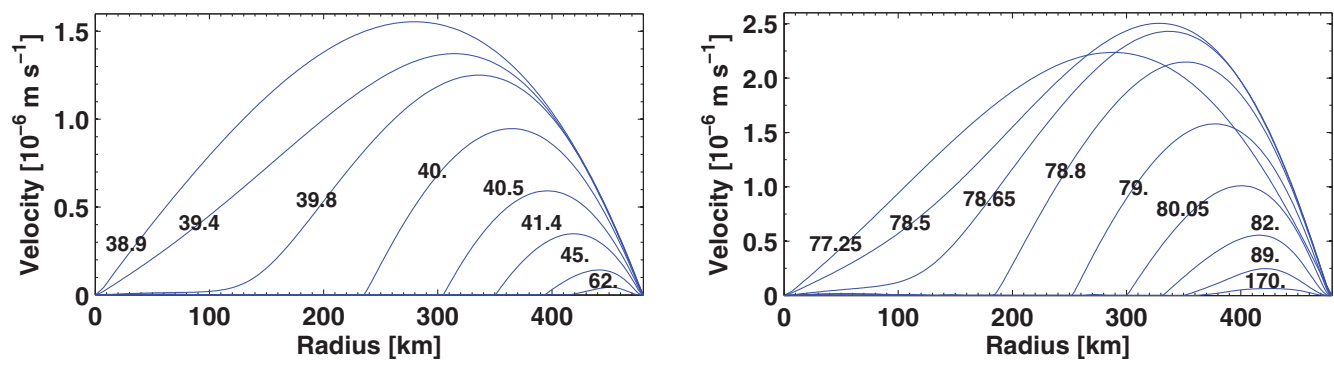

Figure 3. Separation velocity during the differentiation of Ceres. Left panel: Dust density $\rho_{d}=2500 \mathrm{~kg} \mathrm{~m}^{-3}$ (serpentine), initial ice volume fraction $\phi_{0}=0.284$. Right panel: Dust density $\rho_{d}=3600 \mathrm{~kg} \mathrm{~m}^{-3}$ (Vesta-like), initial ice volume fraction $\phi_{0}=0.552$. Further parameters: Grain size $b=0.001 \mathrm{~m}$, water viscosity $\mu_{l}=0.002 \mathrm{~Pa} \mathrm{~s}$, matrix viscosity $\mu_{s}=10^{18} \mathrm{~Pa} \mathrm{~s}$.
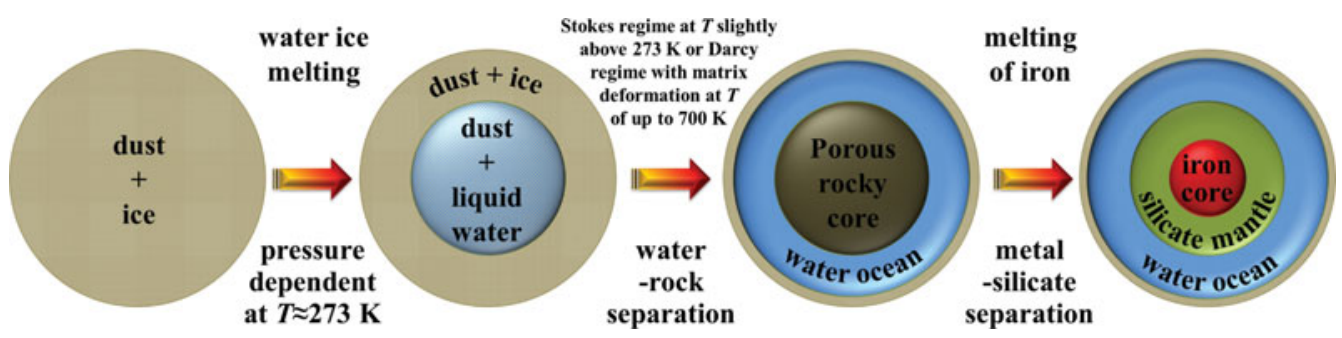

Figure 4. The evolution of Ceres' structure. Early radiogenic heating leads to the melting of ice. Water-rock separation results in a rocky core and water ocean below a solid crust. Further heating leads to the differentiation of a metallic core and a silicate mantle for an early accretion.

Structure evolution of Ceres: The water-rock differentiation occurs early in the evolution of Ceres and should begin during the accretion. An early formation within $\approx 1 \mathrm{Ma}$ after the CAIs results in a rapid temperature increase and an early differentiation. For a later formation time, for instance at $3 \mathrm{Ma}$ after CAIs, the temperature increases slowly, but water melting is still expected within $1 \mathrm{Ma}$ after the formation. In both cases, a water ocean forms fast within $\approx 0.01 \mathrm{Ma}$ after the onset of water ice melting. Subsequent heating of the iron-silicate core depends on the formation time. For an early formation $(1-2$ Ma after CAIs) solidus and even liquidus of metal and silicates is reached within the subsequent $1 \mathrm{Ma}$ and melting is extensive. With a higher concentration of radionuclides in the core after the separation of water, the temperature increases above $2000 \mathrm{~K}$. A complete differentiation into a metal core and a silicate mantle is inevitable, but in such a case the overlying water ocean would boil and evaporate, leading to a dry Vesta-like asteroid. If, however, Ceres forms later (for instance, 3 Ma after CAIs), no metal melt is produced. The ocean contains no heat sources, it is heated by the core from below and isolated by the solid ice-dust crust above. The temperature of the ocean is higher than the melting point of water ice but remains globally below $670 \mathrm{~K}$ (and locally below the evaporation point of water at higher pressures).

\section{Discussion and conclusions}

We modelled the evolution of temperature and the water-rock differentiation for a Ceres-like body using two extreme fractions of ice. We compared theoretical predictions of the separation velocity (Darcy and Stokes law) and, thus, of the timing of the differentiation, with the velocities obtained in two-phase flow calculations. The assumed relative amount of ice (i.e., the density of the silicats, see McCord \& Sotin (2005)) determines the predominant differentiation regime and timing according to Darcy or Stokes law. In the 
Stokes regime, the differentiation proceeds at least three orders of magnitude faster than in the Darcy regime. Both regimes can occur simultaneously in a single body at some intermediate differentiation stages, e.g., Darcy at a depth with a small liquid fraction and Stokes in a water ocean. Using two-phase flow the differentiation is at least one order of magnitude slower than in the Darcy regime. While the relative amount of water determines the differentiation regime (Stokes law should be used for amounts of liquid above a critical fraction for which the rheology of a mixture is dominated by water), two-phase flow calculations should be used instead of Darcy law for small liquid fractions. For the parameters adopted, an early forming Ceres differentiates a water ocean within $\approx 0.01$ Ma. Because we used a lower bound on the matrix viscosity, a slower differentiation is expected for a higher $\mu_{s}$. The temperature evolution indicates metal-silicate separation for an early accretion within the first $3 \mathrm{Ma}$ after CAIs. However, a plethora of additional processes is expected to influence the thermal evolution further. Evolution of the dust porosity, hydration of silicates, convection in the water ocean, for instance, will change the temperature profile in the interior and in the rocky core influencing potential metal differentiation. The interplay of these factors must certainly has been a very complex process that requires further detailed studies.

\section{References}

Šrámek, O., Milelli, L., Ricard, Y., \& Labrosse, S. 2012, Icarus, 217, 339-354

McCord, T. B. \& Sotin, C. 2005, JGR, 110, E05009 